Meta

Journal des traducteurs

Translators' Journal

\title{
Si l'usage est roi, les usagers ne le sont pas...
}

\section{Carlos Del Burgo}

Volume 25, numéro 2, juin 1980

URI : https://id.erudit.org/iderudit/002509ar

DOI : https://doi.org/10.7202/002509ar

Aller au sommaire du numéro

Éditeur(s)

Les Presses de l'Université de Montréal

ISSN

0026-0452 (imprimé)

1492-1421 (numérique)

Découvrir la revue

Citer cet article

Del Burgo, C. (1980). Si l'usage est roi, les usagers ne le sont pas... Meta, 25(2),

258-263. https://doi.org/10.7202/002509ar d'utilisation que vous pouvez consulter en ligne.

https://apropos.erudit.org/fr/usagers/politique-dutilisation/ 


\section{SI L'USAGE EST ROI, LES USAGERS NE LE SONT PAS...}

\section{ÉTAT DE LA QUESTION}

On visera, au cours de cette courte étude, à différencier deux termes que l'usage français tend à confondre. Par ailleurs, on verra que, du côté anglais, il n'existe qu'un équivalent, user, qui se traduit par usager ou utilisateur, selon le sens.

Il apparaît que la polysémie se manifeste surtout dans le domaine des télécommunications.

USER

- (Communications) An individual, installation, or activity having access to a switching center through a local private branch exchange, or by dialing an access code ${ }^{35 *}$.

Ex. : The telephone user can make his own intercom calls $-{ }^{23}$.

- (Automatic data processing) Anyone who requires the use of services of a computing system or its products ${ }^{35}$.

- (Automatic data processing) The person or company using a remote terminal in a time-shared computer system for the purpose of entering a program for execution by the computer ${ }^{40}$.

- user's group : Organizations made up of users of various computing systems to give the users an opportunity to share knowledge they have gained in using a digital computing system and exchange programs they have developed ${ }^{40}$.

* Les numéros indiqués renvoient à la bibliographie. 
- user service (facility) : A user service or facility available on demand to a user and provided as part of a public data network transmission service. Some facilities may be available on a per call basis and others may be assigned for an agreed period at the request of the user ${ }^{24}$ et ${ }^{33}$.

Traduction : service complémentaire offert aux usagers ${ }^{25}$.

- closed user group: A number of users of a public switched data communication service who have the facility to communicate with one another but access is barred to and from all others ${ }^{24}$.

Traductions : groupe fermé d'abonnés ${ }^{24}$. groupe d'usagers fermé ${ }^{31}$.

- user (of the common channel signalling system) ${ }^{33}$.

Traduction : usager (du système de signalisation sur voie commune) ${ }^{25}$.

Voici quelques autres cas de cooccurrence tirés du Ginguay ${ }^{28}$ :

- user : utilisateur, usager, abonné, client;

- user coded: écrit par l'utilisateur (programme, séquence, etc.);

- user coding : séquence(s) écrite(s) par l'utilisateur;

- user controlled: sous la dépendance de l'utilisateur;

- user core : zone utilisateur en mémoire;

- user generated: v. user coded;

- user header label : label début utilisateur;

- user oriented : spécialement conçu pour l'utilisateur, personnalisé ;

- user own coding : v. user coding;

- user supplied: fourni, écrit par l'utilisateur (séquence de programmation, etc.);

- user trailer label: label fin utilisateur;

- user written: v. user coded;

- users association : groupement, association d'utilisateurs;

-users group: v. users association.

USAGER

- Celui qui utilise un service public, le domaine public.

Ex.: Usagers de la route, d'un service public ${ }^{14}$.

- Dans le style administratif, celui qui utilise couramment quelque service public ou quelque moyen de transport, de circulation mis à la disposition du public.

Ex. : Les usagers du chemin de fer, de la route, du téléphone ${ }^{17}$.

- Personne qui utilise habituellement un service public.

Ex. : Les usagers du téléphone. Les usagers de la route, du rail ${ }^{10}$.

- Personnel non informaticien qui utilise, dans le cadre de ses fonctions, les services d'un système ou d'un réseau informatique, en bout de chaîne, par le 
moyen d'un terminal. Par extension, peut qualifier un département ou une entreprise ${ }^{32}$.

Traduction : end-user.

Il s'agit d'une définition rigoureusement identique à celle que donne le même ouvrage du terme «utilisateur».

- Personne d'une entreprise ayant un droit d'usage interne et, par de là même, une responsabilité dans l'exploitation de son poste et la gestion financière des communications émises ${ }^{26}$.

- Groupe d'usagers fermé : closed user group : Un certain nombre d'usagers d'un service public de transmission de données avec commutation ayant la possibilité de communiquer entre eux mais ne pouvant avoir accès aux autres usagers du réseau, ni être atteints par les autres usagers du réseau ${ }^{31}$.

- Ex. : - ces émetteurs offrent désormais aux usagers naviguant dans un rayon de 50 kilomètres la possibilité d'appeler ou de recevoir des appels par le réseau téléphonique national ou international ${ }^{37}$.

\section{UTILISATEUR}

- Personne, appareil, etc. utilisant une chose, etc. ${ }^{29}$

- (Néol.) Personne qui utilise (une machine, un appareil) ${ }^{14}$.

On dira que ceux qui s'opposaient, il n'y a pas si longtemps encore, à l'emploi du terme utilisateur devront se rendre à l'évidence et accepter ce nouveau vocable. Voici d'ailleurs l'opinion de Duprés sur la question : «Utilisateur» est un néologisme bien formé. Il est entré dans notre vocabulaire et personne ne le délogera de la langue courante où il a été précédé par «utiliser» (qui date de 1792), «utilisation» (attesté depuis 1798), «utilisable» (depuis 1842), etc.»

— Qui utilise : les utilisateurs du gaz.

Ex. : Industries utilisatrices du courant électrique 9.

- Personnel non informaticien qui utilise, dans le cadre de ses fonctions, les services d'un système ou d'un réseau informatique, en bout de chaîne, par le moyen d'un terminal. Par extension, peut qualifier un département ou une entreprise ${ }^{32}$.

Il s'agit d'une définition rigoureusement identique à celle que donne le même ouvrage du terme «usager».

Traduction : end-user.

— Ex. : - l'ordinateur est capable de dialoguer en même temps avec plusieurs terminaux, c'est-à-dire de découper son temps de travail entre plusieurs utilisateurs sans que ceux-ci en ressentent de gêne ${ }^{37}$.

- Ex. : - il deviendra de plus en plus nécessaire à l'avenir d'offrir aux utilisateurs et aux prestataires de services le moyen d'établir entre eux, à volonté, des liaisons occasionnelles (Domaine : informatique) ${ }^{37}$.

\section{SYNTHÈSE}

Il ressort des multiples définitions citées que, là où l'anglais ne donne que 
«user», le français établit deux nuances : celle de fréquence d'emploi et celle de nature de l'élément dont on se sert.

En effet, la plupart des dictionnaires français cités s'accordent pour donner à «usager» un caractère usuel, répétitif $10 \mathrm{et} 17$ que «utilisateur» ne possède pas. Par ailleurs, ce dernier terme semble être surtout employé dans le cas d'un appareil, d'un objet, d'une machine (informatique notamment) tandis que «usager» se dit plutôt de quelqu'un qui recourt à un service public ou, du moins, mis à la disposition du public $10,14,15$ et 17 .

Il faut cependant noter que l'usage est, comme d'habitude, capricieux. Effectivement, le Robert-Collins ${ }^{16}$, qui donne d'ailleurs un bon aperçu de la question, traduit user par «utilisateur (machine, dictionary, etc.)» et «usager (public service, telephone, road, train)», mais ne manque pas d'ajouter: "gas users : usagers ou utilisateurs du gaz». On remarquera que la plupart des ouvrages consultés donnent l'expression «utilisateur du gaz», bien qu'il s'agisse d'un service, au même titre que le téléphone, et non d'un objet à proprement parler. À ce sujet, on notera que l'utilisateur du téléphone est celui qui se sert du poste, alors que l'usager du téléphone est la personne, abonnée ou pas, qui recourt avec plus ou moins de régularité au service téléphonique.

Ce qui est vrai en théorie ne l'est cependant pas toujours en pratique. En effet, on retrouve d'abord l'expression usager du poste 26.1 , puis on est plus loin en présence du contexte suivant: «Une liaison spécialisée est une liaison de télécommunications mise à la disposition d'un ou plusieurs utilisateurs sous le régime de la location ${ }^{26.1}$. En téléinformatique, on parle aussi bien d'utilisateurs que d'usagers, que ceux-ci aient affaire à des machines, donc à des objets absolument concrets ${ }^{37}$, ou à des services. Le facteur «fréquence» semble lui aussi ignoré.

On devra donc, face à ce flottement de l'usage, opter systématiquement en faveur du maintien de la double distinction mentionnée précédemment, soit la nature et la fréquence d'emploi de la réalité particulière dont il est question.

Carlos del Burgo

\section{BIBLIOGRAPHIE}

Ouvrages généraux

1. BÉNAC, H., Dictionnaire des synonymes, Paris, Hachette, 1965, 1026 p.

2. DAGENAIS, G., Dictionnaire des difficultés de la langue française au Canada, Montréal, Pedagogia, 1967, 679 p.

3. DAVAU, M., COHEN, M., LALlemand, M., Dictionnaire du français vivant, Paris, (Bruxelles, Montréal), Bordas, 1972, 1338 p.

4. DAVIAULT, P., Langage et traduction, Ottawa, Secrétariat d'État, Bureau fédéral de la traduction, 1962, $397 \mathrm{p}$.

5. DUPRÉ, P., Encyclopédie du bon français dans l'usage contemporain, Paris, Trévise, 1972, 3 vol. 
6. FUNK \& WAGNALLS, éd., Funk \& Wagnalls Standard College Dictionary (Canadian Edition), New York, 1976, 1590 p.

7. GILBERT, P., Dictionnaire des mots nouveaux, Paris, Hachette-Tchou, 1971, 572 p.

8. GIRAUD, J., PAMART, P., RIVERAIN, J., Les Mots «dans le vent », Paris, Larousse, 1971, $251 \mathrm{p}$.

9. LAROUSSE, éd., Grand Larousse Encyclopédique, Paris, 1960-1964, 10 vol.

10. LAROUSSE, éd., Lexis, Paris, $1975,1950 \mathrm{p}$.

11. LAROUSSE, éd., Petit Larousse, Paris, 1978, 1793 p.

12. LITTRÉ, É., Dictionnaire de la langue française, Paris, Gallimard, 1966, 7 vol.

13. MERRIAM-WEBSTER, éd., Webster's Third New International Dictionary of the English Language, unabridged, Springfield (Massachusetts), 1971, 2662 p.

14. ROBERT, P., Dictionnaire alphabétique et analogique de la langue française, Paris, Société du Nouveau Littré, 1960-1964, 7 vol.

15. ROBERT, P., Le Petit Robert ou Dictionnaire alphabétique et analogique de la langue française, Paris, Société du Nouveau Littré, 1978, 2172 p.

16. ROBERT, P., COLLINS, J., Robert-Collins, Dictionnaire français-anglais, anglais-français, Paris, Société du Nouveau Littré, 1978, 1 vol.

17. QUILLET, éd., Dictionnaire encyclopédique Quillet, Paris, 1977, $10 \mathrm{vol}$.

18. THOMAS, A. V., Dictionnaire des difficultés de la langue française, Paris, Larousse, 1974, $435 \mathrm{p}$.

Ouvrages spécialisés

19. BELL CANADA, Services linguistiques, Fichier général, Montréal.

20. BELL CANADA, Service du Trafic, Lexique du trafic; installations, Montréal, 1978, 1 vol.

21. BELL CANADA, Services linguistiques, Lexique général des télécommunications, Montréal, 1979,1 vol.

22. BELL CANADA, Service du Commercial, Lexique général du Commercial, édition revue et augmentée, Montréal, 1978, 1 vol.

23. BELL CANADA, Marketing Department, Manual of Telephone Services (MTS), section 2, p. 1.

24. BELL CANADA, Groupe des communications informatiques, Terminologie de la téléinformatique et des domaines connexes; Computer Communications and Telecommunications Terminology, Ottawa, 1977,73 p. et 32 p.

25. COMITÉ CONSULTATIF INTERNATIONAL TÉLÉGRAPHIQUE ET TÉLÉPHONIQUE (CCITT), Réseaux publics pour données, livre orange, t. VIII. 2, Genève, Union internationale des télécommunications, 1977 , pp. $4-5$ et 146.

26. DUBOIS, M., «Le téléphone : terminologie, équipements et exploitation», in Travail et Méthodes, Paris, 10.77, pp. 15 et 17.

26'. DUBOIS, M., "Télécommunications dans l'entreprise : réglementation", in Travail et Méthodes, Paris 6-7.77, pp. 15 et 19 .

27. Encyclopédie internationale des sciences et des techniques, Vérone, Presses de la cité, 19691972, 10 vol.

28. GINGUAY, M., Dictionnaire d'informatique anglais-français, $4^{\mathrm{e}}$ éd., révisée et complétée, Paris, Masson, 1977, 173 p.

29. GINGUAY, M., LAURET, A., Lexique d'informatique, Paris, 1973, $237 \mathrm{p}$.

30. GRAF, R. F., Radio Shack; Dictionary of Electronics, 4th ed., Forth Worth (Texas), 1975, $688 \mathrm{p}$.

31. GUiLBERT, J.-P., Ouvrage «téléinformatique»; glossaire, s.1., 1976, 40 p.

32. IBM, FRANCE, éd., Terminologie du traitement de l'information/Data processing glossary, Paris, 1977, 154 p. et 145 p 
33. THE INTERNATIONAL TELEGRAPH AND TELEPHONE CONSULTATIVE COMMITTEE (CCITT), Public data networks, orange book, vol. VIII.2, Geneva, International Telecommunication Union, 1977, pp. 4-5 and 146.

34. JAY, F., éd., IEEE Standard Dictionary of Electrical and Electronics Terms, 2nd ed., New York, 1977, $882 \mathrm{p}$.

35. McGRAW-HILL, éd., Dictionary of Scientific and Technical Terms, New York, 1976, 1 vol.

36. POSTES ET TÉLÉCOMMUNICATIONS, éd., Annuaire officiel des abonnés au téléphone de la région de Paris; liste alphabétique, Paris 1977, 2 vol.

37. POSTES ET TÉLÉCOMMUNICATIONS, éd., Documents d'information des Télécommunications, Paris, classement 4 , art. 115, p. 3 ; classement 4 , art. 116, p. 6; classement 8 , art. 118 , p. 1.

38. POSTES ET TÉLÉCOMMUNICATIONS, Précis d'enseignement professionnel; exploitation téléphonique, Paris, Imprimerie Nationale, 1967, $303 \mathrm{p}$.

39. QUILLET, éd., Encyclopédie des sciences industrielles Quillet, Paris, 1973-1974, 4 vol.

40. SIPPL, C. J., SIPPL, C. P., Computer Dictionary and Handbook, Indianapolis (USA), Howard, W. Sams, 1978, 778 p.

41. SMITH, E. C., Glossary of Communications, Chicago, Telephony, 1971, 547 p.

42. TECHNIQUES DE L'INTÉNIEUR, éd., Électronique, t. E6, Paris, 1948, section E 2851. 